\title{
EDUCAR LA RESILIENCIA DESDE LA PINTURA, EL CINE Y EL TEATRO
}

\author{
Carlos Martín Bravo \\ Departamento de Psicología \\ Facultad de Educación de Palencia \\ Universidad de Valladolid \\ loscartinmar@gmail.com
}

Fecha de Recepción: 9 Enero 2019

Fecha de Admisión: 30 Abril 2019

\section{RESUMEN}

"Educar la resiliencia desde la pintura, el cine y el teatro" pretende hacer ver la necesidad de conectar estas disciplinas a problemas complejos. En ese sentido, la presente comunicación parte de la idea de que se puede: A) Educar en resiliencia desde la pintura; para ello se analiza la obra de Géricault "La balsa de la Medusa". B) Educar en resiliencia desde el cine; para ello nos introducimos en el pensamiento de un gran director de cine como es John Ford y en una de sus joyas: "El joven Lincoln". Y C) Educar en resiliencia desde el teatro, para este último menester nos adentramos en una de las mentes más agudas del mundo clásico como es Aristófanes y en su maravillosa obra "Las nubes". Se termina la comunicación con un debate donde se pone de manifiesto la utilidad de las respuestas resilientes a los dramas que se recogen (situaciones de canibalismo, acusación de asesinato y maltrato de mayores) y su utilidad en situaciones actuales.

Palabras claves: resiliencia; drama; "La tabla de la Medusa”; Géricault; "El joven Lincoln”; John Ford; Aristófanes; "Las nubes"; estrategias de afrontamiento

\section{ABSTRACT}

Educate resilience from painting, cinema and theater. "Educate about resilience from painting, film and theater" aims to show the need to connect these disciplines to complex problems. In this sense, the present communication starts from the idea that it is possible to: A) Educate in resilience from painting; we choose Géricault's painting, "The raft of the jellyfish" to analyze. B) Educate in resilience from cinema; this time we introduce the thought of a great film director, John Ford, one of his best movies: "The young Lincoln". And C) Educate in resilience from theater, Aristophanes, a prominent playwright of the classical period, his wonderful work "The cloud" is our chioce. This communication ends up with a debate that highlights the usefulness of resilient responses to the dramas that are collected (situations of cannibalism, accusation of murder and elder abuse) and its usefulness in current situations. 
Keywords: resilience; drama; "The table of the Medusa"; Géricault; "The Young Lincoln"; John Ford; Aristophanes; "Clouds"; coping strategies

\section{OBJETIVOS DE LA INVESTIGACIÓN}

En la presente comunicación se pretende ofrecer una visión de transversalidad entre la educación resiliente y las posibilidades que nos ofrecen campos, tradicionalmente lejanos de la psicología, como son el cine, la pintura, o el teatro. Estamos en tiempo de resiliencia como, quizás, en todos los momentos históricos ha estado presente (Barudy Labrin, 2917). Es por ello que necesitamos buscar formatos nuevos para poder ejecutar conductas resilientes en los distintos escenarios 0 situaciones de la vida. De este modo los objetivos del presente estudio son:

1.- Se puede educar en resiliencia a través de la pintura: "La balsa de la Medusa" de Géricault.

2.- El cine como herramienta de educación resiliente: "El joven Lincoln" de John Ford. 3.Descubrir la educación en resiliencia a través del teatro: "Las nubes" de Aristófanes.

\section{MUESTRA Y PARTICIPANTES}

El estudio que presentamos hace referencia a una serie de parcelas del conocimiento humano (psicología, cine, teatro, pintura...) desde las que se puede analizar cómo el ser humano afronta las situaciones de drama, dolor, en primer lugar, para luego intentar encontrar soluciones regulatorias de esas mismas situaciones anómalas o disruptivas. Situaciones que analizamos para poder encontrar las claves que hagan más soportable y llevadero el dolor humano. Así:

\section{Se puede educar en resiliencia a través de la pintura: La "Balsa de la Medusa" de Géricault.}

Comenzamos, pues, con la pintura. Aquí optamos por escudriñar en un cuadro de Theodore Géricault: "La Balsa de la Medusa”, el cual nos ofrece un amplio abanico de posibilidades analíticas. En dicho cuadro interviene una serie de personajes reales que en la época (1819) fueron muy controvertidos y polémicos por diferentes razones. (Jaubert, 2014; Allen TMusic VEVO, 2012).

Géricault culminó esta oba antes de haber cumplido la treintena, convirtiéndose en un icono del Romanticismo francés. Es una pintura de formato grande $(5 \times 7 \mathrm{~m}$.) que representa una escena del naufragio de la fragata de la marina francesa Méduse, encallada frente a la costa de Mauritania el 2 de julio de 1816. Al menos 147 personas quedaron a la deriva en una balsa construida apresuradamente, y todas ellas, salvo 15, murieron durante los 13 días que se tardó en rescatarlos. Los supervivientes debieron soportar el hambre, la deshidratación, el canibalismo y la locura (Correard y Savigny, 2014). El suceso llegó a ser un escándalo internacional, en parte porque sus causas fueron atribuidas a la incompetencia del capitán que actuaba bajo la autoridad de la reciente y restaurada monarquía francesa.

Figura 1: La balsa de la Medusa de Géricault (1819)

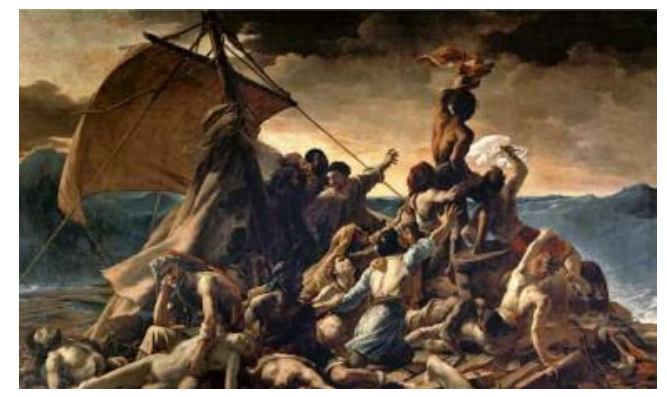


En la obra podemos ver a diversos personajes que reflejan distintas maneras de afrontar el drama del naufragio, es decir, vemos distintos tipos de resilientes. Géricault refleja un hecho que conmocionó a la sociedad francesa de la época y nos hace ver la actitud de cada ser humano ante la adversidad. Todos los personajes del cuadro tienen el mismo problema o viven el mismo drama, pero sus respuestas son distintas. Si nos centramos en el análisis de los personajes que aparecen en el lienzo de Géricault podemos establecer varios grupos de comportamientos que se vinculan a diferentes tipos de resilientes. Así, los cuatro personajes de la base del lienzo han dejado de de tener ninguna actitud de respuesta a la adversidad. Están moribundos y/o en fase de estarlo. El personaje que sentado está con el brazo apoyando su cabeza nos genera una "lectura" de incapacidad ante el drama que se está viviendo. Su posible resiliencia está agonizada. No cree que haya esperanza. Mientras que los personajes con los brazos al viento en la parte alta del cuadro, reflejan que no han perdido la esperanza. Ellos se encuentran en situación de persistencia desesperada que les alimenta una capacidad de afrontar la adversidad.

La presentación de este cuadro el 25 de agosto de 1819 en el Salón de París fue un escándalo. A pesar de su título banal, "Escena de naufragio", y de haber sido colgada muy alto, la obra causó furor. «Interpela y atrae todas las miradas», escribió un periodista. Naturalmente, todos reconocieron la tragedia de La Medusa. Así, y como ejemplo, la presencia de un marinero negro en el centro de la composición también deja patente el compromiso del pintor en un momento en que se intensificaba la lucha contra la esclavitud y la trata de negros. Por su realismo, dramatismo y teatralidad, el lienzo se convirtió en el manifiesto de la pintura romántica, vehículo de pasiones, desesperación y muerte.

La prensa diaria se abalanzó ávidamente sobre el tema. En septiembre de 1816, el «Journal des débats" de París fue el primero en lanzar la terrible noticia sobre el naufragio de la fragata «Medusa». El pintor se propuso hacer una obra realista. Empezó por reunirse con los dos náufragos y trazó los primeros esbozos basándose en sus testimonios. Encargó, curiosamente, una maqueta de la balsa a un carpintero, otro superviviente del naufragio. Dio gran importancia a la representación de los cuerpos. Hizo posar a los supervivientes, a su amigo Delacroix y a su asistente Louis-Alexis Jamar. Para plasmar el color de los miembros amputados y la rigidez de los cadáveres realizó múltiples bocetos en una morgue cercana. Hasta consiguió que un amigo médico le prestara restos anatómicos. Según su biógrafo, Charles Clément, en el taller reinaba una fetidez atroz.

\section{Se puede educar en resiliencia a través del cine: "El joven Lincoln" de John Ford.}

El cine de John Ford, lo mismo que el de Jean Renoir y Alfred Hitchcock tenía que ser interactivo mucho antes de la irrupción de la cultura del videojuego. La conexión del espectador con el cine entendida como un diálogo emocional, películas en las que tengamos que pensar, no consumir pasivamente, sostiene Joseph McBride ${ }^{1}$. Fort Apache me fascinó por su mirada al revisionismo histórico. Yo siempre estuve al lado de los nativos americanos, era algo natural en mí: habiendo sido víctima de bullying de pequeño siempre he tenido tendencia a identificarme con el lado perdedor. En esos años

1 Joseph McBride nació periodista en Wisconsin, paisano de Orson Welles, como le gusta recordar estaba muy implicado en la lucha por los "Civil Rights" y al llegar a casa y ver wésterns en los que los indios siempre perdían me exasperaba. Imagínese lo que fue para mí descubrir un wéstern como Fort Apache en el que los nativos americanos son los buenos y ganan la batalla.

John Ford dijo que estudiar la guerra civil de los Estados Unidos era su primer amor y el cine, el segundo. Para demostrar esta aseveración nos vamos a centrar en una de sus películas emblemáticas: "El joven Lincoln" (Darryl, 1939). Se centra en una época muy específica dentro del "padre 


\section{EDUCAR LA RESILIENCIA DESDE LA PINTURA, EL CINE Y EL TEATRO}

de la patria", y es en el año 1832, donde retrata el perfil de un joven de 23-24 años, leñador y con una habilidad innata de afrontar los problemas (dramas) con el típico humor, casi irlandés. No olvidemos que John Ford procedía de una amplia familia (13 hermanos) de origen irlandés. Lo que nos interesa, en ese caso, es la obsesión, casi clonada de la documentación histórica. John Ford es un revisionista de la historia y para el caso de la película citada, hará una amplia revisión de lecturas y de fuentes fiables como son la obra de Herndon (1892). Gallagher, señala, ya más tarde, (1988) que lo que Ford hace es crear la imagen de América en la figura de Lincoln. Ford es fiel, de manera amplia, a los dos volúmenes de de las memorias de Herndon y no se ha desentendido de los acontecimientos y de los hechos. Ello se pone de manifiesto en la semejanza entre la obra literaria y la obra fílmica. Es por ello que Ford no se aleja de la historia, sino que se sitúa en el punto de encuentro entre el personaje histórico y el mito. (Gutiérrez Delgado, 2011)

Uno de los momentos del drama de la película del "Joven Lincoln" es la tensión que genera cuando el pueblo, en masa, quiere condenar y ahorcar a los dos jóvenes (hermano Clay) que están acusados de cometer un crimen. Es el momento en que el joven abogado se enfrenta al grupo y gestiona con maestría una situación de tensión máxima. Ello supone que a través del juego de las emociones, del humor y de la capacidad de asertividad el jovenzuelo de Lincoln (23 años) comienza a dar respuesta resiliente al drama de la pobre familia Clay. Es una situación histórica que el propio Herndon (1892) recoge en su obra.

Figura: 2 El joven Lincoln (Henry Fonda) logra convencer a la chusma con referencias emocionales de "si es una diversión eso de ahorcar" o, "qué quieren quitarle el primer cliente que va a tener como abogado de la defensa" (ver desde el minuto 31 al 37). Ver: http://cort.as/-GPID

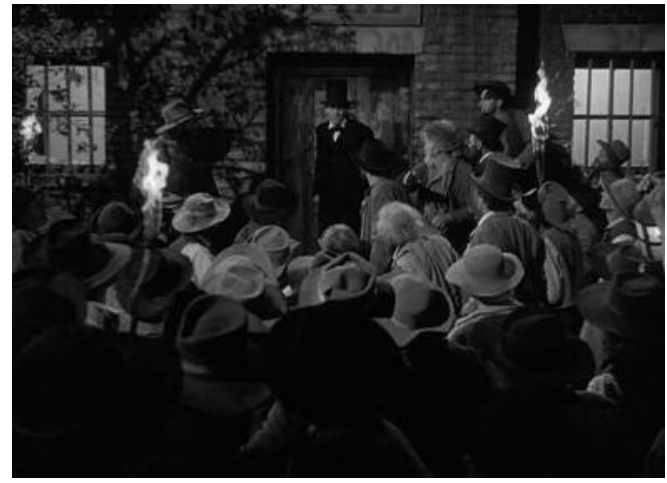

El "Joven Lincoln" es una película entre la leyenda y el documental histórico, con la salvedad de que su historicismo se ve reforzado por la existencia de un documento como el de Herndon y Weik (1892) en el que se constata la fundamentación histórica de la ficción de Ford. Ahora bien, como escribe Merchant (1971) sobre esta película: "supera los límites del realismo", al ofrecer una perspectiva de la historia; es decir, la historia misma y la reinterpretación épica del poeta.

\section{Se puede educar en resiliencia a través del teatro: "Las Nubes" de Aristófanes.}

El teatro es otra de las herramientas que ayudan a soportar los dramas y las situaciones en conflicto. Este es el caso del teatro griego, donde la gente tenía la obligación de ir, mientras que los actores ponían en escena los problemas que se vivían. Es tanta la fuerza terapéutica del teatro que al 
final de la escenificación los ciudadanos de la polis griega se quedaban para debatir sobre los asuntos tratados. Es lo que se denominaba como teatro fórum. De este modo, la función de democracia era puesta en escena. Las Nubes de Aristófanes (2011) podría servirnos de ejemplo.

En las Nubes Aristófanes ${ }^{2}$ critica la degeneración de la educación y la falta de autoridad que convierte a los jóvenes en amantes de los placeres y la buena vida en vez de educarlos en el esfuerzo y la sobriedad. Personaliza todos estos vicios en el personaje de Sócrates y su "pensadero"3 donde alecciona a sus discípulos. También carga contra los especuladores y charlatanes así como contra Ios políticos y abogados que engañan al pueblo. La historia nos habla de Estrepsiades, un padre de origen humilde casado con una mujer de buena familia acostumbrada al lujo y el despilfarro. Su hijo, Fidípides, sólo piensa en los caballos y las carreras y no quiere estudiar. Estrepsiades esta agobiado por las deudas que le producen los costosos vicios de su mujer y su hijo. Entonces tiene la idea de enviar a este último con Sócrates para que le enseñe a vencer con el "argumento débil", esto es, con los trucos que usan los políticos y oradores para ganar las causas cuando no tienen razón. Sócrates aparece rindiendo culto a las nubes, las únicas diosas que considera y que simbolizan la confusión y el engaño de los que se sirve para sus fines en medio de una escuela y unos discípulos que componen un cuadro surrealista propio de los mismísimos hermanos Marx. El chico, aleccionado por estos "profesores" que enseñan a ganar sea como sea los pleitos, acaba pegando, literalmente, a su padre que, enfurecido, quema el "pensadero" de Sócrates.

2 Estamos en el siglo V antes de Cristo. La sátira de Aristófanes es contra los sofistas, dentro de los incluye al "insospechado" Sócrates. Este Sócrates es muy diferente al Sócrates del Protágoras de Platón

3 Palabra inventada por Aristófanes y que tiene un sentido, no obstante, burlón.

Figura 3: Las nubes representan el argumento débil, pues son cambiantes permanentemente. Por el contrario, se presenta el arte de la oratoria como herramienta resiliente

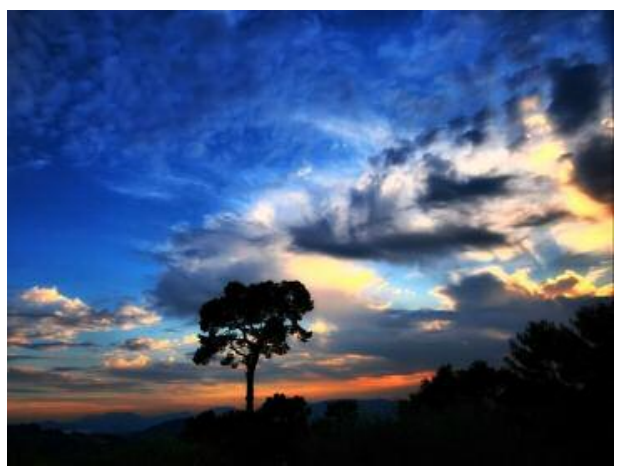

\section{RESULTADOS Y DISCUSIÓN}

\section{El debate del educar la resiliencia desde el arte}

Esto vimos en el enorme cuadro de Géricault el cual nos ayuda a entender diversas maneras de afrontar el drama. De este modo, surgen las siguientes reflexiones:

Recogemos, en este último apartado lo que podríamos llamar un acercamiento al perfil psicología que nos posibilitan las tres referencias resilientes con las que hemos trabajado. En este sentido han trabajado otros autores (Menezes dos Anjos y Morán Astorga, 2016) De este modo, es de 
admirar cómo el joven pintor Géricault se sirve del arte para poder ayudar a los franceses de la época a superar este trauma nacional que supuso la aceptación de la antropofagia. El cuadro es un canto a la resiliencia, pues si lo percibimos como un triángulo, iría desde la base donde aparece la desesperación y la muere, hasta la cúspide donde se recoge la esperanza del náufrago que agita al aire un trozo de tela como reclamo de esperanza. Se podría decir que este lienzo de Géricault refleja la actitud positiva y negativa ante el drama. Es un catálogo de diversos tipos o actitudes resilientes antes el dolor.

\section{El debate del educar la resiliencia desde el cine}

En cuanto al comportamiento resiliente de la histórica figura de Lincoln, se podría reflejar los siguientes rasgos (Reyzábal y Sanz, 2014): Capacidad de afrontamiento, recursos o competencias personales y dureza o resistencia. Es cierto que las grandes figuras de la historia suelen estar dotados de estos tres rasgos. Se cumplen los rasgos citados en los comportamientos del joven Lincoln. Hay, a lo largo de la muy documentada película, situaciones de afrontamiento que demuestran una fortaleza que anula todo tipo de conato de estrés. Ciertamente son recursos psicológicos que ciertos individuos despliegan en situaciones de conflicto o drama. Finalmente, se podría señalar que nuestra figura histórica está dotada de una fortaleza de carácter que podría ser denominada como: "personalidad resistente" (Godoy-Izquierdo y Godoy, 2002). Este rasgo de "personalidad resistente" presenta un perfil en el que se ubican aspectos como: 1.- el compromiso; 2.- el control que viene a ser la capacidad de analizar la situación de conflicto, y, finalmente; 3.- el reto que viene a ser una percepción positiva de los cambios, entendidos no como amenazas, sino como oportunidades.

\section{El debate del educar la resiliencia desde el teatro}

Las comedias griegas ofrecen siempre un abanico de interpretaciones y sugerencias basadas en el delirante ingenio de sus autores y en una libertad de palabra, a menudo políticamente incorrecta, a la altura de la que podemos disfrutar hoy sino mayor. Nosotros hemos querido hacer una interpretación, que probablemente el autor nos habría discutido, basada en el contraste entre la "buena" y la "mala" educación. Criticando la educación como manipulación al servicio de los mercados (Sánchez-Silva, 2019) en la que se fomenta una competitividad para la que lo único que importa es ganar aunque sea usando el "argumento débil", esto es, los trucos y el marketing inherentes a una "sociedad de la imagen" que más lo es de la ilusión o la apariencia, tal como esas nubes que se ven en el cielo tomando diversas formas para engañar nuestros sentidos haciéndonos ver espejismos como los que nos han llevado hasta donde estamos. En todo caso estas obras griegas buscan "sacudir" al espectador haciéndole pensar y tomar partido sobre lo que significa la democracia, la educación, etc.

Así pues, el argumento bueno del malo en la educación se refleja en la sátira del autor griego, cuando al final de la obra hace un alegato de la mala educación que imparte Sócrates que, para Aristófanes, es uno de tantos sofistas. Allí demuestra, por ejemplo, que con falsas argumentaciones lo correcto es que los hijos puedan pegar a sus padres; pues se hace por su bien. Recogemos, seguidamente un fragmento de una escena hilarante sobre este asunto (Aristófanes, 2011):

Fidípides ${ }^{4}$. Reanudo mi discurso en donde tú lo has interrumpido, y principio por preguntarte:

¿Me pegaste cuando era chico?

Estrepsíades. Si, porque te quería y miraba por tu bien.

Fidípides. Dime, ¿no será justo que ahora mire yo igualmente por tu bien, y te pegue, puesto que el pegar a uno es mirar por su bien? ¿Es razonable que tu cuerpo este exento de palos y el mío 
no? ¿No nací yo de tan libre condición como tú? Lloran los hijos, ¿y no han de llorar los padres? ¿Crees que los padres no deben llorar?

Estrepsíades. ¿Por qué?

Fidípides. Tú dirás que la ley tolera que el niño sea castigado, y yo replicaré que los viejos son dos veces niños, y que es más justo castigar a los viejos que a los jóvenes, por cuanto sus faltas son menos excusables.

Estrepsíades. Pero ninguna ley establece que el padre sea castigado.

Fidipides. ¿No era hombre como tú y como yo el que primeramente presentó aquella ley y persuadió a los antiguos a que la aprobasen? Pues bien: ¿qué se opone a que yo haga una nueva por la cual los hijos puedan a su vez castigar a los padres? De buen grado os perdonamos los golpes recibidos antes de la promulgación de esta ley, y consentimos el haber sido maltratados impunemente. Mira cómo los gallos y los demás animales se vuelven contra sus padres; sin embargo ¿se diferencian de nosotros en otra cosa que en no redactar decretos?

Estrepsíades. Ya que imitas a los gallos en todo, ¿por qué no comes estiércol y duermes en un palo?

Fidípides. No es lo mismo, querido; Sócrates no admitiría ese argumento.

Estrepsíades. Porque lo justo es que yo te castigue; y que tú castigues a tu hijo, si alguno te nace.

Fidípides. ¿Y si no me nace? Habré llorado en vano, y tú morirás burlándote de mí.

Estrepsíades. En verdad, amigos míos, voy creyendo que tienes razón, y que se les debe conceder lo que es equitativo. Justo es que seamos castigados si no andamos derechos.

( Ver http://cort.as/-GVcn pp. 100-105).

La herencia del debate político que se genera en el teatro griego de obras como la comentada se refleja en los nuevos escenarios políticos. Somos deudores, en la actualidad, del argumentario justo e injusto que se refleja en la obra de Aristófanes. En este sentido, el papel del político actual no ha cambiado mucho, 0 nada, del papel de los sofistas a los que criticaba Aristófanes hace 25 siglos. Pues como comenta el propio Cyrulnik (2017) en la medida que los políticos escogen "ser" de izquierdas o de derechas dejan de razonar y se dedican a recitar. Palabras como "desaceleración económica" o "no es no" responderían a esta forma de usar estrategias resilientes que es una forma de simplificar la realidad para poder soportarla o manipularla. En esta línea se encuentran pensamientos como los de Kahneman (2000) o Bauman (2010, 2009).

\section{CONCLUSIONES}

En estas tres situaciones observamos distintas formas de afrontar el problema o el drama. En el caso del cuadro ("La balsa de la Medusa") se trata de cómo situarse ante una conducta humana tan oculta en nuestro ser, como es el canibalismo que desplegamos en situaciones de extrema necesidad. La segunda de las situaciones hace

4 Fidípides es el hijo y Estrepsíades el padre referencia a la acusación de asesinato sin pruebas, donde John Ford (director de cine) nos presenta una escena maravillosa, donde un joven abogado, "El joven Lincoln" escenifica magistralmente una práctica de conducta resiliente para superar la terrible tensión que aparece en un fragmento de la película. Y, finalmente Aristófanes, hace 25 siglos y en una obra llena de cinismo nos muestra cómo se puede enseñar el arte de la argumentación para justificar que lo injusto se puede transformar en justo. Así, el joven hijo, que pega a su propio padre y, éste, parece estar de acuerdo. Así pues:

\section{Géricault nos enseña que:}

1.- La resiliencia se puede aprender si logramos empatizar con el dolor ajeno. Es difícil enten- 
der cómo podríamos afrontar el sobrevivir comiendo los cadáveres de los otros.

2.- La resiliencia necesita del "saber esperar". La espera es otro rasgo que se desprende del análisis del cuadro. Los marineros de lo alto que agitan sus ropas para intentar solicitar una ayuda que parece esquiva. Esto hace que buscar y encontrar la ayuda precisa es otra de las claves del resiliente ante el drama.

3.- Educar en resiliencia supone que existe (aunque no lo veamos) la figura del buen samaritano. La espera activa supone no desesperar y entender que en la constancia de persistir está parte del éxito.

4.- En cualquier caso, el debate o la discusión está en hacerse preguntas de gran calado. Así: ¿Si nos tocase vivir una situación similar, qué haríamos? ¿Qué nos ayuda más a salir del drama, nuestra capacidad intelectual o nuestras creencias y emociones? ¿La inteligencia es suficiente para dar respuesta al drama de nuestras vidas?

\section{John Ford nos enseña que:}

1.- Dar soluciones resilientes está en saber mezclar sabiamente el drama con el humor. Se pretende educar a través del cine, donde el papel, en este caso, de Henry Fonda (actor que hace de Lincoln) nos lo posibilita, como se ha visto.

2.- A lo largo de este "biopic" que parte de un drama (asesinato del ayudante del sheriff por parte de unos inexpertos jóvenes, hermanos Clay), John Ford nos presenta momentos sublimes de humor con otros momentos de reflexión. Dicho de otra forma, Ford lograr conectar el humor con la reflexión para afrontar el drama. De tal manera que estos rasgos (humor-reflexión) aparecen más de una docena de veces y que responden claramente a estrategias resilientes.

\section{Aristófanes nos enseña que:}

Educar en el amor al teatro es tanto como decir educar en el amor al debate, a la reflexión y al intercambio. En este punto aparece un elemento potencialmente peligroso como son las redes sociales entendidas como espacio donde no se da la oportunidad al debate, a la reflexión o al intercambio. Si esa condición no se cumple estamos amenazados por un lenguaje totalitario (Cyrulnik, 2016) y esto supone estar amenazados por la confrontación y los dramas. Es, pues necesario educar en la autonomía del lenguaje y de pensamiento para poder afrontar los desajustes y confrontaciones con mayor cantidad de recursos. Y, esto, es tanto como decir que necesitamos una educación resiliente.

\section{REFERENCIAS BIBLIOGRÁFICAS}

Aristófanes (2011). Comedias 2. Madrid: Gredos. Se puede leer "Las nubes" en este URL recortado: http://cort.as/-GVcn

Allen TMusic VEVO (2012). La balsa de la Medusa (Documental). Ver el siguiente URL recortado: http://cort.as/-GT m

Bauman, Z. (2010). Tiempos líquidos: vivir en una época de incertidumbre.

Madrid: Ediciones Tusquets.

Bauman, Z. (2009). Ética posmoderna. Madrid: Siglo XXI.

Barudy Labrin, J. (2917). Tiempos de resiliencia. El País (27 de marzo 2016). Cyrulnik, B. y Anaut, M. (2016). ¿Por qué la resiliencia? Barcelona: Gedisa

Cyrulnik, B. (2017). Conferencia dada en Bogotá. Ver este URL recortado: http://cort.as/-GVgb

Correard, A. y Savigny, H. (2014). El naufragio de la Medusa. Ediciones el viento.

Darryl, F. (productor) y Ford, J. (director) (1939). El joven Lincoln. (Película cinematográfica) EE.UU. 
Century Fox. URL recortado: http://cort.as/-GKxV

Godoy-Izquierdo, D. y Godoy, J. F.( 2002). La personalidad resistente: Una revisión de la conceptualización e investigación sobre la dureza. Clínica y Salud, 13, 2, 135- 162.

Gutiérrez Delgado, R. (2011). Young Mr. Lincoln (1939). El rastro de la historia en el mito de John Ford. Film-Historia XX, 2, 1-24.

Herndon, W.H. y Weik, J.W. (1892): Abraham Lincoln: The True Story of a Great Life. Appleton and Company, New York.

Jaubert, A. (Realizador) (2014). La balsa de la Medusa. Paris: Arte France Palette (production). http://cort.as/-GM1W (URL recortado).

Kahneman, D. (2000). Pensar rápido, pensar despacio. Barcelona: Debate

Menezes dos Anjos, E. y Morán Astorga, C. (2016). A personalidade resiliente: Uma conceptualizaçào teórica. International Journal of Developmental and Educational Psychology. INFAD. Revista de psicología, №1-Vol.2 2016. ISSN:0214-9877. pp: 151-156.

Merchant, P. (1971). The epic, Metheun \& Co. Ltd., London.

Reyzábal,, M. V. y Sanz, A. I. (2014). Resiliencia y acoso escolar. Madrid: La Muralla, S.A.

Sánchez-Silva, C. (2019). La universidad, el último caramelo para la especulación. El País, 7-4-2019. http://cort.as/-Gaw 
